\title{
Study on the effectiveness of fire suppression deluge systems in tunnels
}

Daan Van den Broecke ${ }^{1,2}$, Richard Emberley ${ }^{3}$, Toni Sietz ${ }^{4}$, and Jose L. Torero ${ }^{5}$, Cristian Maluk ${ }^{1}$

${ }^{1}$ School of Civil Engineering, The University of Queensland, Australia

${ }^{2}$ VK Architects \& Engineers, Belgium

${ }^{3}$ Fire \& Rescue Academy, Queensland Fire and Emergency Services, Australia

${ }^{4}$ Department of Mechanical Engineering, California Polytechnic State University, USA

${ }^{5}$ School of Civil, Environmental and Geomatic Engineering, University College London, UK

\section{Corresponding Author}

Advanced Engineering Building, Staff House Road, School of Civil Engineering

The University of Queensland, St Lucia, QLD 4072, AUSTRALIA

\section{Colloquium}

Fire Research 


\begin{abstract}
The required water flow for adequate deluge system operation during a tunnel fire is currently only prescribed by a few regulatory authorities. Car fires are commonly used as references when addressing water-based suppression; nevertheless, limited public information exists on the amount of water required for correctly suppressing a car fire and the thresholds by which the suppression systems fails to be effective. This paper aims at delivering key experimental outcomes to fill this gap in tunnel fire suppression knowledge. The effectiveness of deluge sprinkler systems was investigated by performing a series of full-scale car fire experiments. These experiments were performed by symmetrically positioning a single car under a single sprinkler nozzle, releasing the water flow at a certain time from ignition (which defines the size of the fire), and carefully gauging the burning behaviour of the car fire. A single car was used as it was deemed as the minimum unit for a fire. Temperatures inside, around, and above the car were measured and infrared camera footage was used to gauge flame heights during the experiment. Results from this study yielded two forms of car fire suppression by a deluge system: gradual and instantaneous. A correlation between heat release rate and required water flow for the deluge system is also presented. Outcomes herein show that a water flow per unit area of $6.6 \mathrm{~mm} / \mathrm{min}$ is the minimum water flow required to effectively reduce the temperature in the immediate vicinity of a car fire of different sizes.
\end{abstract}

Keywords: car fire; tunnel fire; deluge fire suppression systems; full-scale fire test; heat release rate.

\title{
Highlights:
}

- A minimum water flow per unit area of $6.6 \mathrm{~mm} / \mathrm{min}$ was found in this study.

- A car fire inside a tunnel may be gradually and instantaneously controlled.

- A deluge suppression system does not instantaneously extinguish a car fire.

- A deluge suppression system reduces the temperature in the vicinity of a car fire.

\section{Funding sources:}

This research study was funded by the Fire Safety Research Engineering Research Group at The University of Queensland, the Queensland Fire and Emergency Services, and the International Master of Science in Fire Safety Engineering supported by the EU Erasmus Mundus programme. 


\section{Introduction}

Increasing urbanization and population growth drives the need for more efficient transportation solutions. In past decades, the world has become more connected through means of communication and mobility. At the same time, environmental and geographical constraints challenge the way transportation evolves. As a way to overcome these hurdles in an efficient manner, tunnels are becoming more present in modern infrastructure developments.

The fire safety strategy in a tunnel presents a challenging engineering problem. The technical challenges lay in assuring (1) paths are provided for safe egress of users, (2) detection systems are effective in minimizing the timeto-detection of a fire, (3) fire suppression systems are effective in controlling (i.e. limiting fire spread) or suppressing a fire that is mainly defined by the dimensions of the tunnel, and the type of vehicles and their cargo, (4) smoke control systems are effective in keeping egress paths clear of smoke (and paths used during firefighters intervention), and (5) structural fire performance can withstand the full duration of the fire (providing robustness to the overall fire safety strategy).

Deluge sprinkler systems, characterized by yielding large water flows and big droplet sizes, are the most common Fixed Fire Fighting System (FFFS) used in tunnel applications; however, very little public data exists that quantifies their effectiveness or provides quantitative information to be used by designers. The required water flow rate to suppress a tunnel fire remains a matter that is not fully resolved and, consequently, creates a large potential benefit from optimizing a costly installation, maintenance, and drainage of deluge systems in tunneling applications.

To the authors' knowledge, two countries currently have prescribed design guidelines for deluge systems in their tunnels as general practice: Japan and Australia. Japan prescribes a water flow per unit area of $6 \mathrm{~mm} / \mathrm{min}$ [1] whereas the Australian requirement is $10 \mathrm{~mm} / \mathrm{min}$ [2]. The substantial difference between these requirements expresses the current design uncertainty. In countries with a limited prescriptive framework regarding deluge systems in tunnels, often, a more conservative water flow is applied.

Japan has by far the most experience with deluge systems; in the fifty years that they have been deploying sprinklers in their tunnels, deluge activation has occurred in 16 instances. None of these fire events escalated to disastrous proportions and the Japanese tunnel authorities are satisfied with the sprinkler performance in tunneling applications [1]. Experience in Australia is scarcer; tunnel designers have been deploying sprinklers in tunnels since 1992. In 2007, the deluge system of the Burnley Tunnel in Melbourne was proven to be successful [5] after a fire event following the collision of four heavy good vehicles and seven light vehicles. 
With fire suppression systems, decisions on operation are many times backed by stories of success as the ones described above. Nevertheless, success is not necessarily clearly defined [3]. In essence, a fire suppression system does not necessarily completely suppress the fire, success is generally achieved when the fire is controlled. If the level of suppression/control is not defined, then the effectiveness of deluge systems in tunnels cannot be quantified. Currently, most of the available public information on the effectiveness of deluge systems in tunnels relates to the ability to protect the tunnel load-bearing lining structure [4]. Available evidence has enabled authorities and designers to conclude that because of its ability to reduce the temperature of the fire, deluge systems can effectively prevent fire-induced concrete spalling of tunnel linings. Despite these favorable conclusions, it is important to consider that analysis of fire-induced structural damage is complex - involving characteristics of the tunnel, characteristics of the fire, and performance of the fire suppression system. Available public data does not allow to deconstruct these relationships and define the role of the suppression system in damage control. Tunneling projects require a more explicit definition of what are appropriate quantitative success criteria for design; current information available does not provide such criteria.

This study aims at providing the basis for experimental data that establishes clear criteria for effectiveness of deluge suppression system design. A single car symmetrically positioned above a single sprinkler nozzle was deemed as the "unit fire", i.e. the least onerous car fire scenario to be addressed. Hence, the objective of this study was to characterize what can be deemed as the absolute minimum required water flow to achieve the fire suppression objective. A series of experiments were conducted to reliably quantify the amount of water a deluge system requires to suppress a car fire in a tunnel and, consequently, provide information for explicit optimization of these fire suppression systems.

\section{Research Methodology}

At the Queensland Fire and Emergency Services (QFES) academy in Whyte Island, Brisbane, Australia, a series of experiments were conducted using cars as units of fuel to characterize the interaction between a deluge water suppression system and a car fire. The car was deemed as the minimum unit of fuel for a tunnel. In the early stages of fire growth, when the deluge system should be activated, the fire remains as a fuel-controlled fire. Nevertheless, heat feedback is likely to enhance the fire during later stages of the fire. For the present experiments heat feedback was ignored so that the least onerous car fire scenario was established, and to investigate the minimum water required to suppress/control such fire. This sets a baseline value that could be refined if more onerous were established. 
Since the deluge nozzles used in tunnels cannot easily be scaled down in all of their features, a common deluge nozzle was placed at a typical, fixed tunnel height. The height was deemed as a minimum height making the nozzle as effective as possible. The fire load consisted of production cars; all typical elements of the fuel load could be taken into account. Specific to car fires, the presence of the roof that does not allow for water to penetrate to the burning areas in an effective manner (e.g. burning car seats). This feature is considered very relevant to the fire scenario, and one that cannot be ignored; therefore, production cars were preferred over an open fire to simulate a car fire.

The experimental setup consisted of a single deluge sprinkler nozzle symmetrically aligned above a car. The positioning of the nozzle, was once again, considered as the ideal location for fire suppression. A steel frame was constructed to position the nozzle at a height of $5.5 \mathrm{~m}$ from the ground and directly above the centerline of the car (see Figure 1).
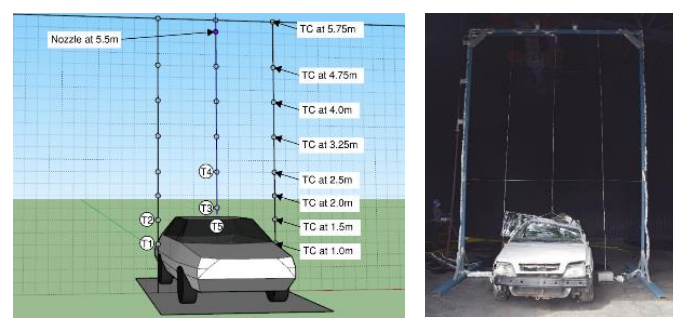

Figure 1 - Schematic and photograph of the fire test setup prior to testing.

\subsection{Instrumentation}

\subsubsection{Thermocouples}

Three thermocouple trees were placed: one at the center and one at each side of the car. The distribution of thermocouples is shown in Figure 1. The thermocouple tree at the center allowed for flame height estimation and assessment of the temperature at different target locations. The thermocouple trees at the sides also enabled to establish the temperatures at the side of the car with the objective of quantifying the potential for fire spread to other vehicles before and after nozzle activation.

Additionally, four thermocouples were placed inside the car: (1) under the roof centred in the car, (2) between the front seats at the handbrake, (3) in the middle of the rear foot compartment, and (4) in the middle of the backseat. The ignition source was placed in the middle of the rear foot compartment. The thermocouple at this location was used to determine the moment of ignition. The choice of ignition location aimed at yielding a car fire that was well protected by the enclosure of the car. A bag of diesel saturated woodchips was used as the source of ignition. This ignition source was placed in the middle of the foot compartment of the backseat. The characterization of the car 
fire by means of thermocouples was mainly to establish average interior temperatures within the car but also to establish the timeline for fire growth.

\subsubsection{Video and infrared cameras}

Video and infrared cameras were used throughout the tests. The video cameras enabled understanding the different stages of the burning behaviour and allowed for gauging of flame heights during periods of sufficient visibility. During stages of poor visibility, the IR camera was the sole source to determine flame height. The facilities at QFES academy do not count with heat release rate measurement provisions, therefore, the heat release rates were inferred from flame height measurements.

\subsection{Water distribution test}

A BETE nozzle type N9W20.4 was used in a single-nozzle configuration as explained above. The water flow $(\dot{V})$ from the nozzle was directly controlled by regulating the water pressure of the system. Water flow from the nozzle is expressed by the following equation.

$\dot{V}=K * \sqrt{p}$

Where, $p$ is the pressure in bar and $K$ is the factor correlating the system pressure with the water flow. A K-factor of $294 \mathrm{l} / \mathrm{min} . \mathrm{bar}^{1 / 2}$ was reported by the manufacturer for the nozzle used in this study. The water flow rate relationship for the BETE nozzle type N9W20.4 is shown in Figure 2. This water flow is distributed over a floor area; governed by the following conditions: water pressure, height of the nozzle, and spray angle. 


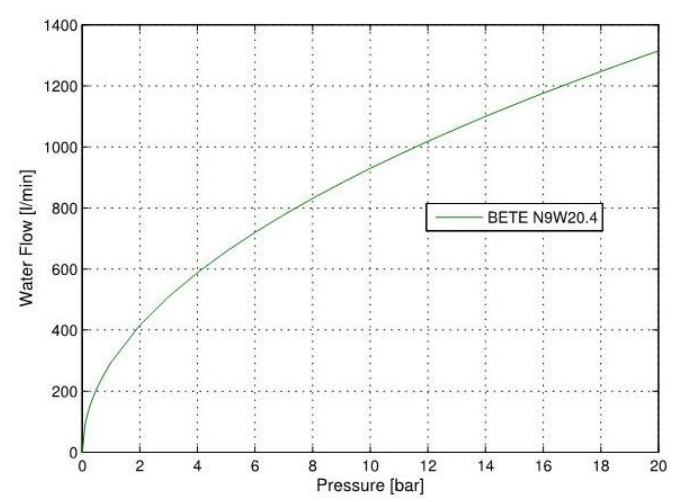

Figure 2 - Pressure - water flow relationship

In order to determine the distribution of the water flow at ground level, a series of standard 'pan tests' were performed, based on the procedure described in UL199 [6]. During these tests, square pans were placed along the radius away from the sprinkler nozzle (see Figure 3). After a certain discharge time, the water flow at these discrete points away from the sprinkler head can be derived by measuring the height of water in each pan and dividing it by the discharge time. The pan test shown in Figure 4 consists out of a strict procedure to ensure consistency. First, a plastic sheet was placed on the boxes while the system activates. In this manner, any distortion during ramp-up of the system pressure was avoided. After stabilization at the requested system pressure, the sheet was removed.

The square pans (with a surface area of $356 \times 356 \mathrm{~mm}$ ) collected dispersed water during a fixed time-period after which the boxes were covered again and the water was turned off. The volume of water in each of the boxes was measured, divided by the surface area of the box, and divided by the time-period; hence the water distribution was quantified as a rate[mm/min]. This process was repeated for a range of operational water pressures (see Figure 3).

In order to assess the radial symmetry of the water distribution, additional pan tests were performed in $45^{\circ}$ increments; this was done at water pressures of 6 and 7 bar. In order to plot the results of the radial water distribution at floor level, the values for the water distribution between the discrete measurement points from the pan tests were interpolated. The resulting 2D plane with the water flow in $\mathrm{mm} / \mathrm{min}$ is shown in Figure 4 below. This plot leads to the conclusion that radial symmetry can be assumed for the purpose of these experiments [7]. 


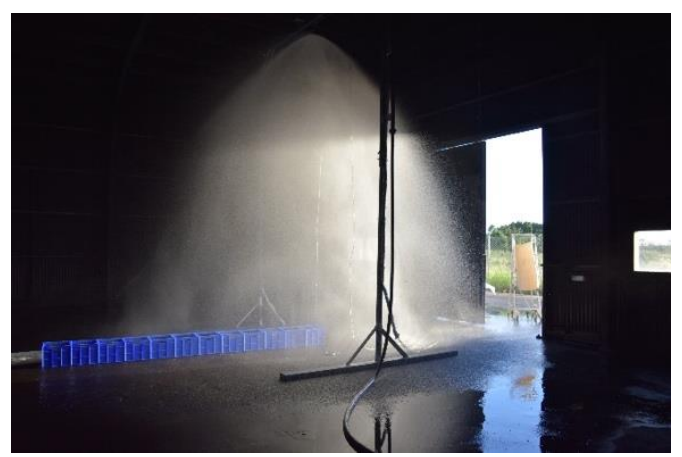

Figure 3 - Setup of the standard 'pan tests' for investigating water distribution from the nozzle. Blue square pans where positioned as shown the photograph.

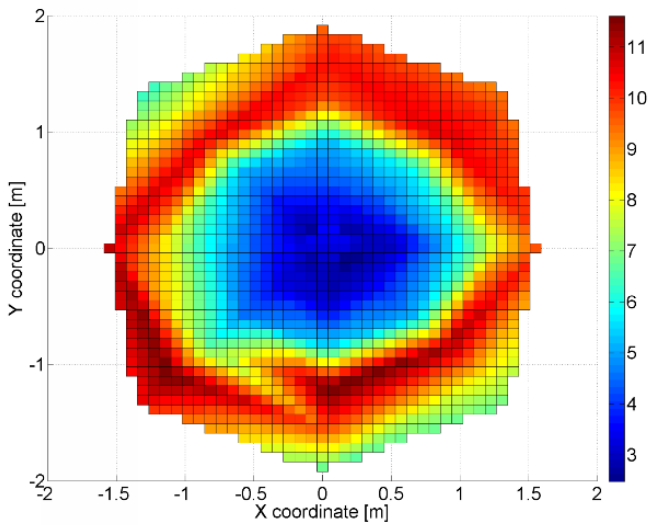

Figure 4-2D water flow distribution at ground surface area for 6 bar.

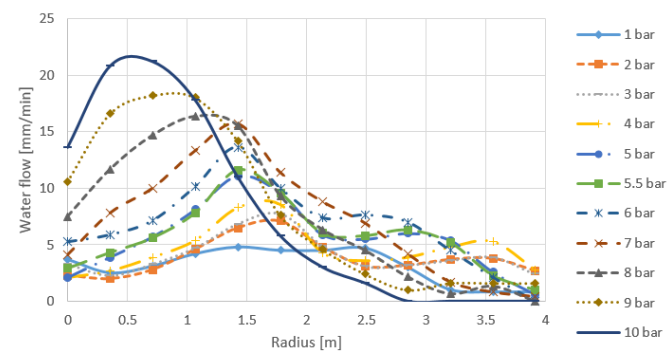

Figure 5 - Water distribution away from the deluge nozzle.

\subsection{Car fire tests}

The main fuel load in cars are the seats, interior lining, plastics in the finishing or the bodywork, the tires, and the fuel tank from the car. Due to safety considerations, fuel tanks were emptied. The absence of fuel in the tank was deemed to not alter the conclusions of this study because during a fire, the tank would likely be breached before activation of the deluge system and also because even if it was, it would result in a larger fire. Given that the aim 
of this study was to provide the minimum realistic water flow to control a fire, a larger fire would not affect the estimation of this minimum value. In case the fire barrier between the engine and the passenger compartment failed, additional fuel from cabling and other combustibles from the engine compartment would be added to the fuel load. The doors of the test cars were removed prior to the test but placed inside the car to maintain the same fuel load. This allowed for more air entrainment to the car compartment, leading to a more rapid-fire growth compared to that of a car with doors intact.

\subsubsection{Heat release rate}

In order to estimate the HRR based on measurements of flame height, the mean flame height was measured. Careful averaging is necessary since the intermittent part of the flame fluctuates several times per second and an instantaneous analysis would therefore not provide a reliable estimate of the heat release rate. The mean flame height is calculated by collecting the flame height of 100 IR video frames (i.e. 4 seconds of IR recording at 25 FPS) and developing a cumulative normal distribution. The mean flame height is the height that corresponds with a presence probability of 0.5 . The camera saturation thresholds were carefully evaluated to demonstrate that the choice of threshold had no effect on the estimate of the flame height.

The SFPE Handbook of Fire Protection Engineering [8] provides Heskestad's flame height correlation. This is the method used to derive heat release rates from the mean flame heights for this study. This correlation is given in equation 2, where $\mathrm{D}$ is the diameter of the fire and $\dot{Q}$ is the heat release rate.

$L=0.235 Q^{2 / 5}-1.02 D$

Rearranging this equation, the heat release rate can be expressed as:

$\dot{Q}=((L+1.02 D) / 0.235)^{5 / 2}$

For equation 3, flame length (L) is derived from the IR camera data and the diameter of the fire (D) is assumed to be equal to the width of the car. 


\section{Test Results}

This section presents an overview of the most significant experimental outcomes of the work described herein. Mainly, the results from the water distribution and car fire tests are shown. Thermocouples T1-T5 (see Figure 1) are the main source of data to characterize the behavior of the car fires.

\subsection{Water Distribution}

As shown in Figure 3, for the 0 to $1 \mathrm{~m}$ radius interval, the water flow for 1 bar is in fact higher than that of 2 and 3 bar. For a water pressure of 1 bar, low flows created an inadequate water spray; as a consequence, a significantly larger amount of water fell closer to the centerline than is the case for 2 bar. The BETE datasheet [9] describes a minimal system pressure of 3.45 bar, therefore, any pressure lower than that should not be used. These measurements served to confirm this guideline. For all fire tests, the nozzle was operated at pressures higher than 3.45 bar.

The variation of the water dispersion along the radius away from the sprinkler nozzle is shown in Figure 5. For a water pressure higher than 7 bar, a single water flow peak occurs away from the nozzle. For lower water pressure the water distribution is distributed with no clear single peak. The variation along the radius away from the nozzle confirms that the distribution of water at the floor area cannot be gauged simply by dividing the water flow rate by the coverage area [10].

An average water flow per unit area rate was established to enable a comparison between the different fires. For this purpose, the floor surface area of a car was assumed to be equivalent to a floor surface of $2 \mathrm{~m}$ diameter circle. The average water flow was therefore defined as the integral of the curves in Figure 3 over a $1 \mathrm{~m}$ radius. This value was used as the average water flow per unit area $[\mathrm{mm} / \mathrm{min}]$. The result of this integration is shown in Figure 6 .

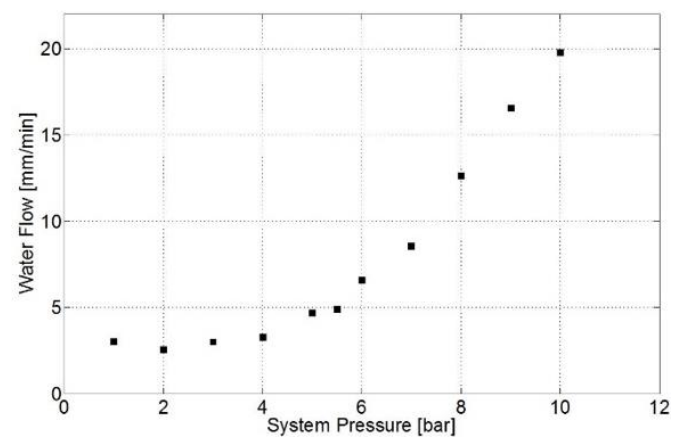

Figure 6 - Representative water flow for the coverage area. 
Figure 7 shows the burning car right before the deluge activation. The dimensions of the representative car are given. In every experiment, the middle section of the car was aligned with the thermocouple tree. For each test, the flames reached past the roof of the car and, therefore Equation (2) was assumed to be valid. The typical sequence of events during a car fire test are:

1. The car fire was ignited as explained in Section 2.2;

2. The fire was allowed to grow, uncontrolled until the interior car temperature reached flashover levels (at about $800-1000^{\circ} \mathrm{C}$ ) before the deluge system was activated;

3. The deluge system was activated at a low range water pressure for an appropriate period of time (see Section 3.2.1);

4. Temperature evolutions for gauges inside the car and in the vicinity ${ }^{1}$ of the car were evaluated during the time in which the suppression system remained active (see Section 3.2.1);

5. The water flow was halted to allow for the car fire to regain size (and temperature) prior to deluge activation;

6. Deluge system was activated at a higher range water pressure for an appropriate period of time (see Section 3.2.1);

7. Steps 4 to 6 were repeated until the car fire reached burnout.

Given that all cars and fires were different establishing a systematic criterion that allowed defining opening and closing of the sprinklers was not possible. Each experiment allowed different observations so each experiment was described individually. The observations were fundamental to the understanding of the impact of the sprinkler on the fire.

The evolution of the temperature as a function of time revealed important features of the behavior of the car fires when different water flows were applied. Arbitrary thresholds were used to characterize different levels of control. These are not fundamental criteria but just simple practical ways of establishing the influence of the water flow on the car fire.

\footnotetext{
${ }^{1}$ The vicinity of the car is defined as thermocouples T1-T4 (Figure 1)
} 
- $\quad$ Temperature drops instantly - a temperature decrease, where all thermocouples in the vicinity of the car recorded a temperature below $100{ }^{\circ} \mathrm{C}$ in less than 100 seconds from the moment of activation. This threshold also includes cases where the inertia induced by the steam pushed down the flame and increased the temperature next to the car before dropping instantaneously as well. Basically, this effect delays the instantaneous temperature decrease next to the car.

- $\quad$ Temperature drops gradually - a temperature decrease, where all thermocouples recorded a temperature below $100^{\circ} \mathrm{C}$ in not less than 100 seconds from the moment of deluge activation.

- $\quad$ Temperature rises - a temperature increase, where all thermocouples recorded a temperature increase to 100 ${ }^{\circ} \mathrm{C}$ or more after 100 seconds from the moment of deluge activation. This criteria was considered as a failure to suppress/control.

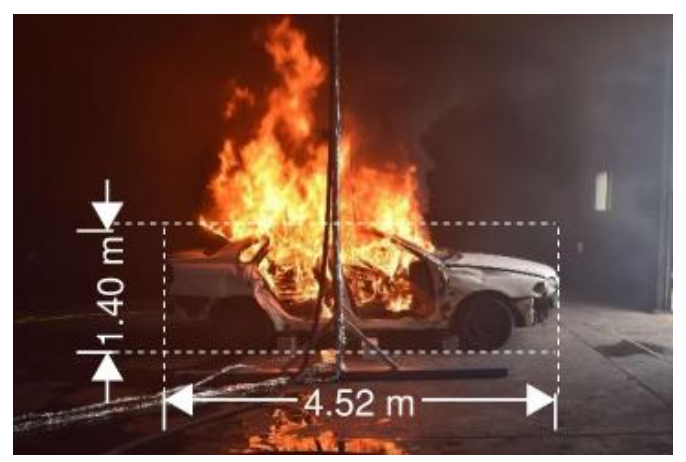

Figure 7 - Typical car fire test (prior to activation of the deluge system)

\subsubsection{Car Fire Test \#1 - Volvo S40 Jul '96}

During the first deluge activation at $2.5 \mathrm{~mm} / \mathrm{min}$, no clear drop in temperatures occurred throughout deluge activation. The temperatures right next to the car rose significantly (Figure 8) resulting in a failure to suppress/control.

The second deluge activation at $8.6 \mathrm{~mm} / \mathrm{min}$ showed a different sprinkler effect: during the 100s of deluge activation, the temperatures around the car dropped to a uniform $50^{\circ} \mathrm{C}$ (Figure 8). The decrease in temperature occurred within these 100s and was therefore assumed as an instantaneous drop. 


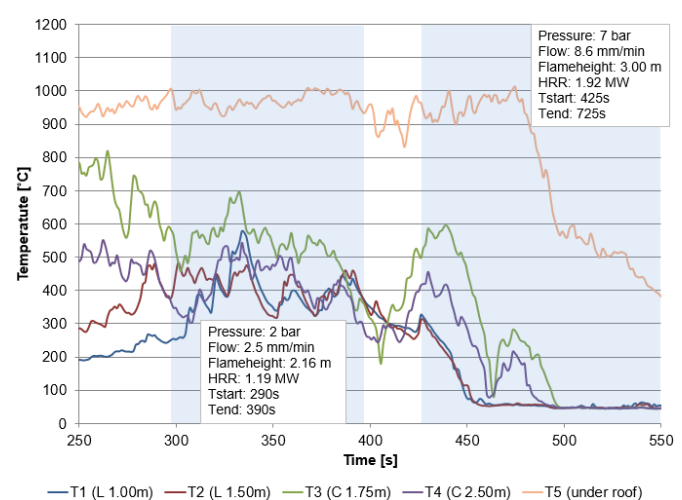

Figure 8 - Temperatures readings during Car Fire Test \#1.

\subsubsection{Car Fire Test \#2 - Saab 9000 CD Jun '94}

The first deluge activation at $3.3 \mathrm{~mm} / \mathrm{min}$, showed the temperature above the car rising continuously (Figure 9).

The fire was therefore not contained inside the car, but the temperatures adjacent to the car were kept under $100^{\circ} \mathrm{C}$.

The second deluge activation at $6.6 \mathrm{~mm} / \mathrm{min}$ resulted a gradual drop in temperature directly above the car (Figure 9).

The third activation of $8.6 \mathrm{~mm} / \mathrm{min}$ showed an instantaneous drop in temperature (Figure 9). An initial rise in temperature right next to the car did occur after deluge activation likely linked with the downward momentum generated by the deluge system, initially pushing the flames down- and out-wards.

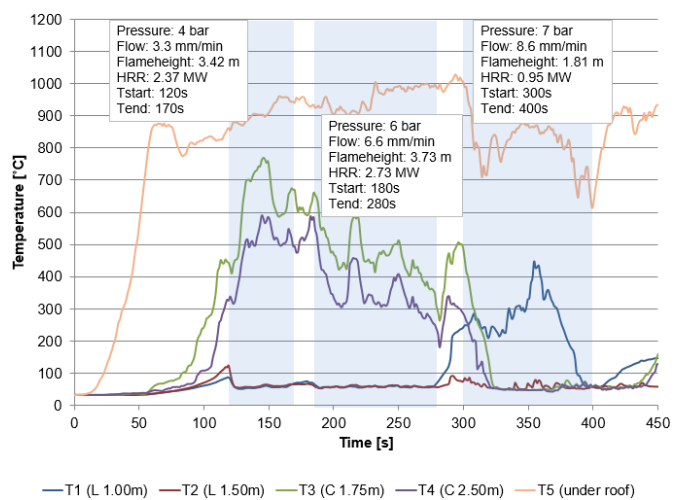

Figure 9 - Temperatures readings during Car Fire Test \#2.

\subsubsection{Car Fire Test \#3 - Honda Civic GL Sep '91}

The first deluge activation at $4.7 \mathrm{~mm} / \mathrm{min}$ showed the fire could not be controlled. The fire grew throughout sprinkler activation (Figure 10); however, the deluge system managed to shield the thermocouples right next to the car from the heat. 
The second deluge activation at $14.1 \mathrm{~mm} / \mathrm{min}$ showed an instantaneous drop in temperature occurred within seconds (Figure 10).

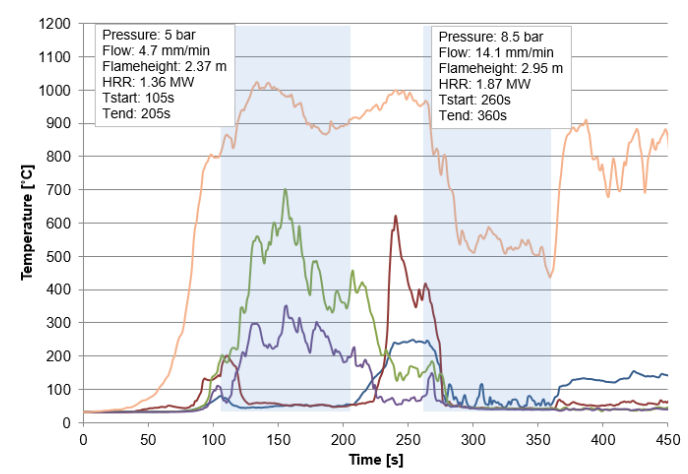

- T1 (L 1.00m) - T2 (L 1.50m) - T3 (C 1.75m) - T4 (C 2.50m) - T5 (under roof)

Figure 10 - Temperatures readings during Car Fire Test \#3.

\subsubsection{Car Fire Test \#4 - Mazda 323 Aug '92}

The first deluge activation at $4.7 \mathrm{~mm} / \mathrm{min}$ showed a decrease of temperature readings in the vicinity of the car fire

(Figure 11); however, a temperature rise occurred above the car within 100s of deluge activation.

The second deluge activation at $14.1 \mathrm{~mm} / \mathrm{min}$ showed a clear instantaneous drop in temperature.

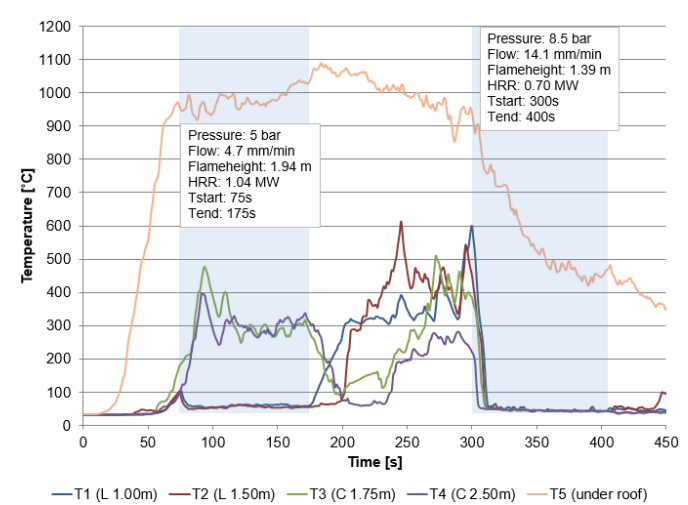

Figure 11 -Temperatures readings during Car Fire Test \#4.

\subsubsection{Car Fire Test \#5 - Holden VT Station Wagon Dec '99}

The first deluge activation at $4.7 \mathrm{~mm} / \mathrm{min}$ was triggered during the early stage of the fire. Early activation was done for this test to extend the range of heat release rates. Temperatures in the vicinity of the car were kept well below $100^{\circ} \mathrm{C}$ (Figure 12). A key observation in this test was the temperatures inside the car continued to increase 
during active suppression, evidencing that the fire was actively contained inside the car and underlines the importance of a fast activation time to avoid fire spread to other cars in the vicinity.

The second deluge activation at $8.6 \mathrm{~mm} / \mathrm{min}$ did not yield any differing results from the first activation, whereas the third activation showed an instantaneous drop in temperatures. Note, an initial rise in temperature right next to the car occurred.

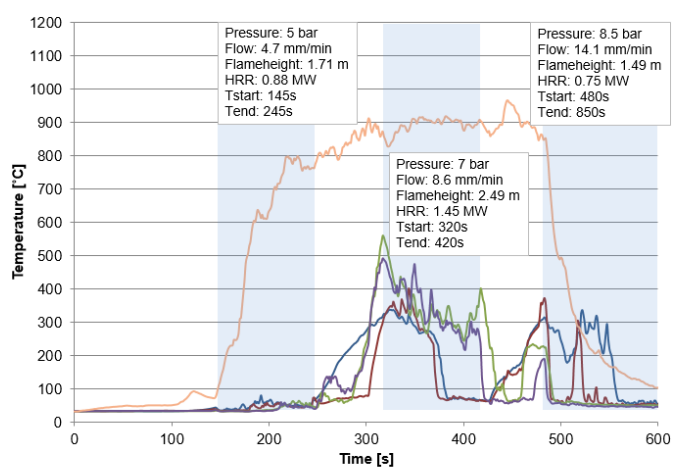

- $\mathrm{T} 1(\mathrm{~L} 1.00 \mathrm{~m})-\mathrm{T} 2(\mathrm{~L} 1.50 \mathrm{~m})-\mathrm{T} 3(\mathrm{C} 1.75 \mathrm{~m})-\mathrm{T} 4(\mathrm{C} 2.50 \mathrm{~m})-\mathrm{T} 5$ (under roof)

Figure 12 - Temperatures readings during Car Fire Test \#5.

\section{Analysis and discussion}

The above criterion for suppression - instantaneous drop, gradual drop and temperature increase was applied to all experiments. Figure 13 is the result of applying this classification on the experimental results.

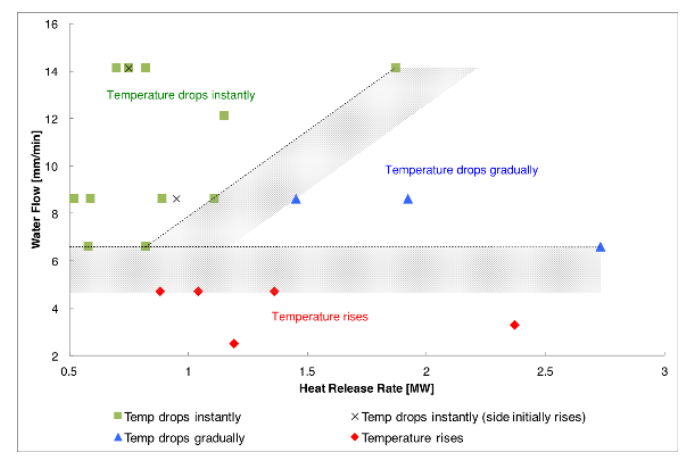

Figure 13 - Plot representing effectiveness of water flow for a range of car fires HRR.

The data points corresponding to the three main categories appear to roughly correspond to the three regions in Figure 13. However, the data point density is insufficient to categorize the plot in three distinct regions divided by lines. The transition from one threshold to another is therefore marked with shaded regions. The most conservative approach to these shaded areas is to assume that the most detrimental scenario would occur in this particular region 
i.e. a temperature rise in the horizontal shaded bar and a gradual rise in the diagonal region. The thresholds between these three regions cannot be determined more accurately than the shaded areas from the given set of data.

The results show that above $6.6 \mathrm{~mm} / \mathrm{min}$ the water flow decreased the temperatures in the vicinity of the car fire. Whether this decrease in temperature is instantaneous or gradual, depends on the water flow that is applied. Essentially, two ways exist for achieving an instantaneous drop in temperature: increase the water flow or limit the HRR of the fire. The latter corresponds to reducing the activation time. However, this is not always possible given the sequence of events that need to occur in an operating tunnel from the moment of detection to activation. In the previous discussion, the temperatures in the vicinity of the car were assessed and led to a description of possible beneficial effects of deluge systems. A temperature reduction in areas in close proximity to the fire is highly relevant for tunnel fire safety design because the potential for fire spread is addressed. This aspect, nevertheless, requires a more comprehensive study.

It is worth highlighting that because of the the specific characteristics of a car firs, the presence of the car roof that does not allow for water to penetrate to the burning areas in an effective manner (e.g. burning car seats). Therefore, mechanisms of fire control or suppression are only by means of watering areas in the vicinity of burning fuel.

\section{Concluding remarks}

A series of five full-scale fire tests were conducted in order to assess the required deluge sprinkler water flow per unit area for suppression of car fires. The results showed that fire suppression deluge systems can effectively reduce the temperatures in the vicinity of a car fire - serving the purpose of controlling fire spread by keeping the fire in the car of origin.

Three suppression thresholds were identified: (1) temperature dropping instantaneously, (2) temperature dropping gradually, and (3) temperature rises continuously. This last criterion resulting in a car fire neither being suppressed nor controlled.

In some cases, where temperature dropped instantaneously, the temperatures in the vicinity of the car fire increased before eventually decreasing sharply as well. This effect is likely to be associated with the water steam generating a downward momentum and effectively pushing the flame down- and out-wards.

An instantaneous drop in temperature was achieved by either applying a sufficiently high-water flow or achieving deluge activation when the fire is relatively incipient. The former requires a system that provides higher water flow rates whereas the latter is achieved by minimizing the activation time - emphasizing the importance of rapid deluge activation. 
The boundaries between the three thresholds depend on the applied water flow. A water flow per unit area of the order of $6.6 \mathrm{~mm} / \mathrm{min}$ managed to drop the temperatures in the vicinity of the car fire. Water flow rates per unit area below $6.6 \mathrm{~mm} / \mathrm{min}$ never controlled/suppressed the car fire. An important note of this study is that the results are only valid for the exact configuration as tested, with no ventilation and for the given fire sizes and with a specific sprinkler head (BETE nozzle type N9W20.4). Additional analysis on scalability is required to investigate the applicability of other cars and scenarios; nevertheless, this scenario is deemed as representing the minimum requirements expected for car tunnels.

\section{Acknowledgments}

The authors would like to acknowledge the Queensland Fire and Emergency Services for the time, help, and resources provided for the experimental phase of the project. The authors would also like to thank the following people for their contributions: Jeronimo Carrascal, John Nguyen, Melinda Trezise, Bronte Nagy-Cox, Tam Do, Andy Wong, Pratvi Patel, Aidon Browning, Angela Solarte, Aaron Bolanos, Michael Conway, Toni Sietz, Shane Gilchrist, Nick Agnew, and Stacey Agnew.

\section{References}

[1] Stroeks, R. Sprinklers in japanese road tunnels. Tech. rep., Ministry of Transport, The Netherlands, 2001.

[2] Bilson, M., Purchase, A., and Stacey, C. Deluge system operating effectiveness in road tunnels and impacts on operating policy. In 13th Australian Tunnelling Conference Proceedings (Melbourne, May 2008).

[3] Carvel, R. Mitigation of tunnel fires. In Proceedings from the Fifth International Symposium on Tunnel Safety and Security (ISTSS 2012) (March 2012).

[4] SOLIT2. Engineering Guidance for a Comprehensive Evaluation of Tunnels with Fixed Fire Fighting Systems. SOLIT2 Project, 2012.

[5] Carvel, R., and Ingason, H. SFPE Handbook of Fire Protection Engineering, fifth edition ed., vol. 3. Springer, 2016, ch. Fires in Vehicle Tunnels, pp. 3303-3325.

[6] Standard for Automatic Sprinklers for Fire-Protection Service, UL 199, 2005.

[7] Van den Broecke, D, Study on the effectiveness of Sprinklers in Tunnels. Master thesis submitted in the International Master of Science in Fire Safety Engineering, The University of Queensland, St Lucia, Australia, 2016.

[8] Heskestad, G. SFPE Handbook of Fire Protection Engineering, fifth edition ed., vol. 1. Springer, 2016, ch. Fire Plumes, Flame Height, and Air Entrainment, p. 402.

[9] BETE N Spray Nozzles with FM Approval, 2016. Available at www.bete.com/pdfs/BETE_N_FM_metric.pdf [Accessed at 1 Jul. 2017] 
[10] Wu, D., Guillemin, D., and Marhsall, A. 2007. A modeling basis for predicting the initial sprinkler spray. Fire Safety Journal. 42 (4) 283-294 